\title{
Incidencia del síndrome postcolecistectomía en pacientes adultos
}

\author{
Incidence of postcholecystectomy syndrome in adult patients
}

\author{
Karla Erika Aldama López, ${ }^{*}$ Paolo Alberti Minutti, ${ }^{\ddagger}$ Ricardo Sanabria Trujillo§ \\ Citar como: Aldama LKE, Alberti MP, Sanabria TR. Incidencia del síndrome postcolecistectomía en \\ pacientes adultos. Acta Med. 2021; 19 (1): 61-66. https://dx.doi.org/10.35366/98572
}

\section{Resumen}

Introducción: La colecistectomía laparoscópica (CL) es el tratamiento ideal para la colelitiasis, pero la persistencia de síntomas postoperatorios es frecuente, a éstos se les conoce como síndrome postcolecistectomía (SPC) y está poco reportado en México, por lo que el objetivo del estudio es determinar su incidencia. Material y métodos: Estudio observacional transversal. Se analizaron 220 expedientes clínicos de pacientes colecistectomizados en 2017, en el Hospital Nuevo Sanatorio Durango. Se evaluaron los cinco síntomas más frecuentes: dolor abdominal en cuadrantes superiores, náusea, vómito, diarrea y distensión abdominal, prequirúrgicamente y a un año de la cirugía. Resultados: El SPC se presentó en $23.1 \%$ (n = 36), los síntomas persistentes fueron: distensión abdominal (21.8\%), dolor (14.7\%), náusea (5.8\%), diarrea (4.5\%) y vómito $(0.6 \%)$. Los pacientes con dolor abdominal de tipo biliar, náuseas y vómito redujeron significativamente al año de la cirugía. La cirugía no modificó el riesgo de presentar diarrea y fue un factor de riesgo para presentar distensión abdominal (OR 2.08). Diez pacientes no tuvieron un diagnóstico certero como causa del SPC (32.3\%). Conclusión: EI SPC se presenta frecuentemente, debe evaluarse de manera correcta y descartar complicaciones postquirúrgicas. Se ha relacionado una mayor incidencia de síndrome de colon irritable tras una CL.

Palabras clave: Colecistectomía laparoscópica, síndrome postcolecistectomía, síndrome intestino irritable, dolor abdominal, distensión abdominal.

\section{Abstract}

Introduction: Laparoscopic cholecystectomy $(\mathrm{CL})$ is the optimal treatment of cholelithiasis, but the persistence of symptoms is frequent and it's called postcholecystectomy syndrome (SPC). In this study, we aimed to determine the incidence of this syndrome. Material and methods: An observational transversal study was performed, 220 clinical records from adult patients who had been done CL in 2017 at the Hospital Nuevo Sanatorio Durango were analyzed. It has been evaluated the five most frequent gastrointestinal symptoms: right upper quadrant abdominal pain, nausea, vomiting, diarrhea and abdominal distension, before and a year after the surgery. Results: SPC was present in $23.1 \%$ of cases $(n=36)$, the persistent symptoms were: abdominal distension $(21.8 \%)$, pain (14.7\%), nausea (5.8\%), diarrhea $(4.5 \%)$ and vomiting $(0.6 \%)$. The number of patients reporting biliary pain, nausea and vomiting reduced significantly over a year of the surgery. The surgery didn't modify the risk of presenting diarrhea, but it is a risk factor to present abdominal distension (OR 2.08). 10 patients had no final diagnosis (32.3\%). Conclusion: SPC is frequent, patients should be carefully examined to exclude postoperative complications. $\mathrm{CL}$ has been associated with increased incidence of irritable bowel syndrome.

Keywords: Laparoscopic cholecystectomy, postcholecystectomy syndrome, irritable bowel syndrome, abdominal pain, abdominal distension.
* Médico Residente $4^{\circ}$ año, Cirugía General, Facultad Mexicana de Medicina de la Universidad La Salle México. Nuevo Sanatorio Durango. Ciudad de México, México.

* Médico adscrito de Medicina Interna, UMAE Hospital de Especialidades, Centro Médico Nacional Siglo XXI, IMSS. Ciudad de México, México.

$\S$ Médico adscrito de Cirugía General, Nuevo Sanatorio Durango. Ciudad de México, México.
Correspondencia:

Dra. Karla Erika Aldama López

Correo electrónico: aldamakarla@gmail.com

Aceptado: 04-06-2020.

www.medigraphic.com/actamedica 


\section{INTRODUCCIÓN}

La colecistectomía es la cirugía gastrointestinal más realizada en México, ${ }^{1}$ se realiza en $95 \%$ por colelitiasis, ya que es una de las patologías gastrointestinales más comunes en el mundo con una prevalencia de $14.3 \%,{ }^{2}$ aunque la mayoría son asintomáticos, algunos pueden llegar a presentar complicaciones como pancreatitis, piocolecisto, perforación vesicular, colecistitis aguda, colangitis o ictericia obstructiva. ${ }^{3}$ Las colecistectomías han ido en aumento desde la llegada de la laparoscopia por presentar mejores resultados en dolor postoperatorio, estancia intrahospitalaria y tiempo de recuperación, ${ }^{4-6}$ además de complicaciones de $0.50 \%{ }^{7}$

A pesar de que se estima que la colecistectomía es exitosa en mejorar los síntomas en $90 \%,{ }^{8}$ existe un porcentaje variable de sujetos que continúan con síntomas gastrointestinales a pesar de la cirugía, ya que se ha descrito que los pacientes postcolecistectomizados tienen mayor cantidad de síntomas gastrointestinales que el resto de la población, ${ }^{9}$ principalmente dolor abdominal en cuadrante superior derecho y dispepsia con o sin ictericia, náusea, distensión abdominal, cambios en los hábitos intestinales y flatulencias, que pueden variar en duración desde los dos días hasta los 25 años después de una colecistectomía. A estos síntomas se les conoce como síndrome postcolecistectomía. ${ }^{9-12}$

En México no se cuentan con estudios al respecto, por lo que el objetivo de este trabajo es determinar la incidencia del síndrome postcolecistectomía en pacientes adultos sometidos a una colecistectomía laparoscópica.

\section{MATERIAL Y MÉTODOS}

Estudio observacional transversal. Se analizaron los expedientes clínicos de los 220 pacientes sometidos a colecistectomía laparoscópica, desde el 1 de enero de 2017 al 31 de diciembre de 2018, en el Hospital Nuevo Sanatorio Durango, se evaluaron los cinco síntomas más frecuentes en la literatura: dolor abdominal en cuadrantes superiores, náusea, vómito, diarrea y distensión abdominal, prequirúrgicamente y a un año de la cirugía.

Se recabaron los siguientes datos en la nota de ingreso: edad, sexo, síntomas prequirúrgicos (dolor abdominal, náusea, vómito, diarrea, distensión abdominal) establecido de acuerdo con la escala visual análoga (EVA) del cero al 10 según la intensidad; tiempo de evolución de dichos síntomas; presencia de comorbilidades gastrointestinales prequirúrgicas y si la colecistectomía laparoscópica fue electiva o de urgencia, según datos clínicos de agudización, así como el diagnóstico prequirúrgico. Por último, se realizó una consulta al año siguiente de su cirugía en la cual se interrogó si había padecido dolor abdominal, náusea, vómito, diarrea o distensión abdominal.

Estos datos fueron recabados con el instrumento de recolección y posteriormente vaciados en una base de datos en Excel, se analizaron en el programa estadístico Statistical Package for Social Sciences (SPSS), versión 24.

Además, se realizó un análisis descriptivo, las variables cualitativas se expresaron en número de eventos y porcentajes, las variables cuantitativas se expresaron conforme a pruebas de normalidad de Kolmogórov-Smirnov, en la que si el comportamiento de la variable era de libre distribución se utilizó mediana y rango intercuartílico, y si era normal, se realizó media y desviación estándar. Se usó la prueba McNemar y t de Student para muestras pareadas.

\section{RESULTADOS}

Se analizaron 220 expedientes en total, se excluyeron 64, ya que dos contaban con historia de malignidad abdominal; cinco fueron colecistectomías parciales; una lesión de vía biliar; tres cirugías laparoscópicas fueron convertidas a abiertas y 53 no contaban con su expediente completo. Se incluyeron en el estudio 156 expedientes de pacientes sometidos a colecistectomía laparoscópica. La edad media fue de 51.8 años con una desviación estándar de 12.77. Con un total de 113 (72.4\%) mujeres y $43(27.6 \%)$ hombres. Los diagnósticos que indicaron la colecistectomía están descritos en la tabla 1. En 118 de los pacientes (75.6\%) la colecistectomía laparoscópica se realizó de forma electiva y en 38 (24.4\%) de urgencia.

\section{Síntomas preoperatorios}

El síntoma preoperatorio más frecuente fue el dolor en cuadrantes superiores $(82 \%, n=128)$, posteriormente náusea $(48.7 \%$, $\mathrm{n}=76)$, vómito $(33.3 \%, \mathrm{n}=52)$, distensión abdominal (13.5\%, $\mathrm{n}=21)$ y el menos frecuente fue la diarrea $(1.9 \%, \mathrm{n}=3)$. En cuanto a la intensidad de dolor preoperatorio se encontró que la mayor frecuencia fue el dolor moderado (5-7 en EVA) en 74 pacientes $(47.4 \%, n=74)$, seguido de dolor severo en EVA mayor de siete $(19.2 \%, \mathrm{n}=30)$, sin dolor $(17.3 \%, \mathrm{n}=27) \mathrm{y}$ dolor leve en EVA menor de cinco $(15.4 \%, \mathrm{n}=24$ ) (Tabla 1).

\section{Síntomas postoperatorios}

El síntoma postoperatorio más frecuente fue la distensión abdominal (21.8\%, $\mathrm{n}=34$ ), seguido de dolor moderado $(7 \%, n=11)$ y leve $(6.4 \%, n=10)$, náusea $(5.8 \%, n=9)$, diarrea $(4.5 \%, \mathrm{n}=7)$, dolor severo $(1.3 \%, \mathrm{n}=2)$ y vómito $(0.6 \%, \mathrm{n}=1$ ) (Tabla 1).

La distensión abdominal postquirúrgica se presentó en 34 pacientes (21.8\%), acompañado de dolor en 25 de 
Tabla 1: Características generales $(\mathrm{N}=156)$.

\begin{tabular}{|c|c|}
\hline & n (\%) \\
\hline Pacientes femeninos, $\%$ & 72.4 \\
\hline Edad, media $\pm \mathrm{DE}$ & $51.8 \pm 12.77$ \\
\hline \multicolumn{2}{|l|}{ Diagnóstico prequirúrgico } \\
\hline Coledocolitiasis & $2(1.3)$ \\
\hline Pancreatitis aguda & $7(4.5)$ \\
\hline Colelitiasis asintomática & $24(15.4)$ \\
\hline Colecistitis crónica litiásica & $82(52.5)$ \\
\hline Colecistitis crónica litiásica agudizada & $41(26.3)$ \\
\hline \multicolumn{2}{|l|}{ Indicación quirúrgica } \\
\hline Electiva & $118(75.6)$ \\
\hline Urgencia & $38(24.4)$ \\
\hline \multicolumn{2}{|l|}{ Síntomas preoperatorios } \\
\hline Sin dolor & $27(17.3)$ \\
\hline Dolor leve & $24(15.4)$ \\
\hline Dolor moderado & $74(47.4)$ \\
\hline Dolor severo & $30(19.2)$ \\
\hline Náusea & $76(48.7)$ \\
\hline Vómito & $52(33.3)$ \\
\hline Diarrea & $3(1.9)$ \\
\hline Distensión abdominal & $21(13.5)$ \\
\hline Asintomática & $26(16.6)$ \\
\hline \multicolumn{2}{|l|}{ Síntomas postoperatorios } \\
\hline Sin dolor & $133(85.3)$ \\
\hline Dolor leve & $10(6.4)$ \\
\hline Dolor moderado & $11(7.0)$ \\
\hline Dolor severo & $2(1.3)$ \\
\hline Náusea & $9(5.8)$ \\
\hline Vómito & $1(0.6)$ \\
\hline Diarrea & 7 (4.5) \\
\hline Distensión abdominal & $34(21.8)$ \\
\hline Asintomática & $120(76.9)$ \\
\hline Más de un síntoma & $25(16.0)$ \\
\hline
\end{tabular}

ellos (73.5\%). De los pacientes cuyo único síntoma fue la distensión abdominal uno reingresó por coledocolitiasis residual (2.9\%) y uno con síndrome de colon irritable (2.9\%) diagnosticados por el servicio de gastroenterología. Once de los pacientes con distensión abdominal no tuvieron un diagnóstico final (32.3\%).

Siete pacientes tuvieron diarrea $(12.5 \%)$, seis de ellos (85.7\%) también presentaron distensión abdominal, sólo un paciente (14.2\%) cursó únicamente con este síntoma, fue estudiado y posteriormente se diagnosticó con colitis ulcerativa por colonoscopia. Las náuseas se presentaron en nueve pacientes $(5.7 \%)$, se encontró siempre ser concomitante con distensión abdominal. Sólo hubo un paciente con vómito postquirúrgico, acompañado de distensión y atribuido a síndrome de intestino irritable diagnosticado por gastroenterología.

El número de pacientes con dolor abdominal de tipo biliar, las náuseas y el vómito redujeron significativamente al año de la cirugía; sin embargo, la cirugía no modificó el riesgo de presentar diarrea, pero fue un factor de riesgo para presentar distensión abdominal (OR 2.08, $\mathrm{p} \leq 0.05$ ) (Tabla 2).

Acerca del dolor postoperatorio a un año, $85.3 \%(\mathrm{n}=$ $133)$ del total de pacientes no presentó dolor, $7 \%(n=11)$ tuvo dolor moderado y $6.4 \%(n=10)$ leve, manteniendo a dos pacientes $(1.3 \%)$ con dolor severo que ameritaron reingreso a urgencias con diagnóstico de síndrome de intestino irritable tras seguir el protocolo diagnóstico específico por gastroenterología (Tabla 1).

El dolor fue el síntoma con mayor mejoría a un año de la cirugía pasando de una media prequirúrgica en EVA de 5.13 a una media postquirúrgica de 0.69 en EVA ( $p \leq$ 0.001) (Tabla 3).

Los pacientes que se mantuvieron asintomáticos durante el año de la colecistectomía laparoscópica fueron 120 (76.9\%). Existieron cuatro pacientes que fueron reingresados de urgencia, tres de ellos por coledocolitiasis residual y uno por absceso residual (Tabla 4).

Tabla 2: Síntomas reportados en el prequirúrgico y a un año de la colecistectomía laparoscópica.

\begin{tabular}{|c|c|c|c|c|c|}
\hline & Prequirúrgico & A un año & OR & IC 95\% & $p$ \\
\hline Dolor biliar & 128 & 23 & 0.028 & 0.006 a 0.083 & $<0.001^{*}$ \\
\hline Náusea & 76 & 9 & 0.056 & 0.015 a 0.151 & $<0.001^{*}$ \\
\hline Vómito & 52 & 1 & 0.019 & 0.001 a 0.112 & $<0.001^{*}$ \\
\hline Diarrea & 3 & 7 & 3.0 & 0.53 a 30.3 & 0.288 \\
\hline Distensión & 21 & 34 & 2.08 & 1.01 a 4.55 & $0.048^{*}$ \\
\hline
\end{tabular}


Tabla 3: Intensidad del dolor

prequirúrgico y postquirúrgico.

\begin{tabular}{lcccc} 
& \multicolumn{3}{c}{ Número } & \\
Dolor & Media & de casos & DE & $p$ \\
\hline Prequirúrgico & 5.13 & 156 & 2.97 & $<0.001^{*}$ \\
Postquirúrgico & 0.69 & 156 & 1.77 & \\
\hline
\end{tabular}

DE = desviación estándar.

Se usó la prueba t de Student para analizar cambios de la intensidad del dolor.

* Significativo $p<0.05$.

El síndrome postcolecistectomía se presentó en 36 pacientes $(23.1 \%)$, cuatro pacientes con causas biliares consecuencia de la cirugía (11.1\%) y síntomas gastrointestinales extrabiliares en 32 pacientes (88.9\%). Además, $69.4 \%$ de estos pacientes ( 25 pacientes) tuvieron más de un síntoma (Tabla 4).

Veintitrés pacientes (14\%) tuvieron dolor crónico después de la colecistectomía, 22 de ellos (95.6\%) se acompañaron de distensión abdominal, los diagnósticos finales de pacientes con dolor abdominal a más de un año se representan en la tabla 5 .

\section{DISCUSIÓN}

La colecistectomía es la cirugía gastrointestinal más frecuente en México, ${ }^{1}$ es el estándar de oro del tratamiento para la colelitiasis. ${ }^{3}$ Se ha descrito que causa una mejoría en aproximadamente $90 \%$ de los pacientes; ${ }^{10}$ sin embargo, pueden recurrir síntomas similares a los experimentados antes de la cirugía.

En nuestro estudio, la persistencia de la mayoría de los síntomas del SPC fue de $23.1 \%$ (36 pacientes) y de estos pacientes $69.4 \%$ (25 pacientes) tuvieron dos o más síntomas, esto es similar a lo que describe la literatura, ya que el SPC se reporta de 12 a $47 \%$. $^{13,14}$

Además, $11 \%$ de estos pacientes tuvo una causa biliar del síndrome y $86.1 \%$ con causas extrabiliares no relacionadas con la cirugía, por esta razón el síndrome postcolecistectomía siempre debe ser investigado para descartar un origen biliar. ${ }^{15}$

A diferencia de la literatura, en el que el síntoma principal fue la diarrea, ${ }^{16}$ seguida de dolor en cuadrantes superiores y flatulencias, ${ }^{17}$ en este estudio el síntoma más frecuente fue la distensión abdominal seguido de dolor abdominal, náusea, diarrea y, por último, vómito.

De los cinco síntomas gastrointestinales evaluados, los que llamaron la atención fueron diarrea (presente en 4.5\% de todos los pacientes) y distensión abdominal (21.8\%), ya que éstos mostraron mayor incidencia únicamente de forma postoperatoria, con una prevalencia similar al de la literatura de $0.9-35 \% ;_{;}^{14}$ sin embargo, la cirugía no modificó el riesgo de presentar diarrea, pero ésta se muestra como un factor de riesgo para presentar distensión abdominal (OR 2.08, p $\leq$ 0.05).

La prevalencia de la diarrea postcolecistectomía se ha reportado de 0.9 a $35.6 \%$, es más común en pacientes que no siguen un régimen dietético bajo en grasa, con una incidencia de $5.7 \%$ a los tres meses de postoperados aproximadamente; la población con más tendencia a sufrir este síntoma son hombres menores de 45 años y se ha sugerido una relación con el trastorno de ansiedad. ${ }^{14}$ Es un síntoma común postcolecistectomía que ha mostrado mejoría con colestiramina. ${ }^{18-20}$

El síntoma que mostró mayor mejoría fue el dolor en cuadrantes superiores, ya que $76.9 \%$ de los pacientes se encontraron asintomáticos a un año de la cirugía, esto es similar a la bibliografía. ${ }^{21}$ Cabe mencionar que alrededor de $10 \%$ de los pacientes colecistectomizados regresan por dolor, ${ }^{22}$ y de ellos aproximadamente $5 \%$ no tienen una causa identificada, ${ }^{23,24}$ en este estudio $8.7 \%$ de los pacientes que tuvieron dolor postoperatorio crónico no tuvieron un diagnóstico final.

\begin{tabular}{|cc|}
\multicolumn{2}{c}{ Tabla 4: Presencia de síndrome } \\
postcolecistectomía (N = 36). \\
\hline \\
n (\%) \\
\hline Biliares & $4(11.1)$ \\
Coledocolitiasis residual & 3 \\
Absceso residual & 1 \\
Extrabiliares & $31(86.1)$ \\
Más de un síntoma & $25(69.4)$ \\
\hline
\end{tabular}

\section{Tabla 5: Diagnósticos finales de pacientes con dolor crónico > 1 año ( $\mathrm{N}=23)$.}

\section{$\mathrm{n}(\%)$}

Síndrome de colon irritable

$16(69.6)$

Gastritis crónica

$2(8.7)$

Enfermedad por reflujo gastroesofágico

$1(4.3)$

Coledocolitiasis residual

$1(4.3)$

Colelitiasis asintomática

$24(15.4)$

Sin diagnóstico final 
Los síntomas de dispepsia preoperatorios y una historia crónica de dolor abdominal, así como cólicos vesiculares son predictores potenciales de pobres resultados y persistencia del dolor tras una colecistectomía. ${ }^{21}$

Se ha descrito que los pacientes colecistectomizados tienen un riesgo de 2.2 veces mayor (IC 95\%, $p=0.03$ ) de padecer síndrome de intestino irritable que los no colecistectomizados, ${ }^{25}$ y se ha asociado de forma fisiopatológica al flujo de una concentración alta de ácidos biliares hacia el colon, lo que provoca diarrea en algunos pacientes, ${ }^{26}$ también se ha descrito un componente emocional, ya que se presenta en pacientes ansiosos o deprimidos. ${ }^{13}$

Los síntomas de dispepsia no específica y distensión abdominal no son indicaciones suficientes para realizar una colecistectomía, porque éstos no se verán afectados después de la cirugía. ${ }^{13}$

En México en 2016 se realizó un estudio con 345 pacientes que padecían síndrome de colon irritable con el subtipo de predominancia de diarrea, en el cual se describió que la prevalencia de dicho síndrome en pacientes colecistectomizados fue 3.6 veces mayor. ${ }^{27}$

Por otro lado, la incidencia de gastritis diagnosticada por endoscopia por dolor crónico postcolecistectomía también se ha descrito antes, ${ }^{28}$ se considera a la colecistectomía como un riesgo significativo para la gastritis biliar, ${ }^{29}$ causante de reflujo y cambios en la mucosa gástrica, ${ }^{30}$ incluso se ha supuesto tratamiento con ácido ursodesoxicólico en el tratamiento para la gastritis por reflujo alcalino postcolecistectomía con efectos positivos. ${ }^{31}$

De hecho, se ha sugerido que en pacientes que serán sometidos a una colecistectomía deba realizarse una endoscopia preoperatoria, ya que en $28.8 \%$ de los casos existen cambios endoscópicos que expliquen el dolor abdominal, ${ }^{32}$ los diagnósticos más frecuentes causantes del síndrome postcolecistectomía son úlceras pépticas y hernias hiatales. Cabe mencionar que mientras más tarde en presentarse el dolor después de una colecistectomía, menos probable es que se relacione con ésta. ${ }^{33}$

Las limitantes en este estudio incluyen la falta de evaluaciones prequirúrgicas específicas del síndrome de intestino irritable según criterios de Roma IV, así como un diagnóstico endoscópico para determinar si estas patologías estaban presentes antes de la colecistectomía.

\section{CONCLUSIONES}

En este estudio la frecuencia del síndrome postcolecistectomía se presentó en $23.1 \%$ de los casos. Al ser una entidad frecuente todos los pacientes con el síndrome postcolecistectomía deben ser estudiados para descartar otras posibles causas; en primer lugar se deben descartar complicaciones postquirúrgicas de origen biliar como coledocolitiasis o abscesos residuales. Todos los síntomas postquirúrgicos deben ser evaluados correctamente.

Aunque la colecistectomía laparoscópica se considera efectiva por causar una mejoría clínica en aproximadamente $90 \%$ de los pacientes, ${ }^{10,34}$ también se ha relacionado con una mayor incidencia de distensión abdominal incluso considerándola como factor de riesgo de desarrollo de algunas patologías como gastritis biliar o síndrome de colon irritable de predominio diarrea. ${ }^{25,29}$

Existe un porcentaje variable en el que los síntomas postcolecistectomía no tienen un diagnóstico certero como causa.

\section{REFERENCIAS}

1. División Técnica de Información Estadística en Salud. El IMSS en cifras. Las intervenciones quirúrgicas. Rev Med Inst Mex Seguro Soc. 2005; 43 (6): 511-520.

2. Enríquez-Sánchez LB, García-Salas JD, Carrillo-Gorena J. Colecistitis crónica y aguda, revisión y situación actual en nuestro entorno. Cir Gen. 2018; 40 (3): 175-178.

3. Duncan CB, Riall TS. Evidence-based current surgical practice: calculous gallbladder disease. J Gastrointest Surg. 2012; 16 (11): 2011-2025.

4. García RA, Gutiérrez RL, Cueto GJ. Evolución histórica de la cirugía laparoscópica. Rev Mex Cir Endoscop. 2016; 17 (2): 93-106.

5. Chousleb E, Chousleb A, Shuchleib S. Estado actual de la colecistectomía laparoscópica. Revista de gastroenterológica mexicana. Rev Gastroenterol Mex. 2004; 69 (Supl. 1): 28-35.

6. Memon MA, Deeik RK, Maffi TR, Fitzgibbons RJ Jr. The outcome of unretrieved gallstones in the peritoneal cavity during laparoscopic cholecystectomy. A prospective analysis. Surg Endosc. 1999; 13 (9): 848-857.

7. MacFadyen BV Jr, Vecchio R, Ricardo AE, Mathis CR. Bile duct injury after laparoscopic cholecystectomy. The United States experience. Surg Endosc. 1998; 12 (4): 315-321.

8. Vander Velpen GC, Shimi SM, Cuschieri A. Outcome after cholecystectomy for symptomatic gall stone disease and effect of surgical access: laparoscopic v open approach. Gut. 1993; 34 (10): 1448-1451.

9. Wanjura V, Sandblom G. How do quality-of-life and gastrointestinal symptoms differ between post-cholecystectomy patients and the background population? World J Surg. 2016; 40 (1): 81-88.

10. Jaunoo SS, Mohandas S, Almond LM. Postcholecystectomy syndrome (PCS). Int J Surg. 2010; 8 (1): 15-17.

11. O'Rourke NA, Jansen A. Post cholecystectomy symptoms. In: Cox M, Eslick G, Padbury R editors. The management of gallstone disease. New York, USA: Springer International Publishing; 2018. pp. 205219.

. Ros E, Zambon D. Postcholecystectomy symptoms. A prospective study of gall stone patients before and two years after surgery. Gut. 1987; 28 (11): 1500-1504.

13. Luman W, Adams WH, Nixon SN, Mcintyre IM, Hamer-Hodges D, Wilson $\mathrm{G}$ et al. Incidence of persistent symptoms after laparoscopic cholecystectomy: a prospective study. Gut. 1996; 39 (6): 863-866.

14. Yueh TP, Chen FY, Lin TE, Chuang MT. Diarrhea after laparoscopic cholecystectomy: associated factors and predictors. Asian J Surg. 2014; 37 (4): 171-177.

15. Schofer JM. Biliary causes of postcholecystectomy syndrome. J Emerg Med. 2010; 39 (4): 406-410. 
16. Manríquez E, Tejos R, Rojas A, Pimentel E, Vega T, Achurra P et al. Diarrea poscolecistectomía: ¿un problema frecuente? Rev Chil Cir. 2017; 69 (5): 376-381.

17. Lamberts MP, Lugtenberg M, Rovers MM, Roukema AJ, Drenth JP, Westert GP et al. Persistent and de novo symptoms after cholecystectomy: a systematic review of cholecystectomy effectiveness. Surg Endosc. 2013; 27 (3): 709-718.

18. Arlow FL, Dekovich AA, Priest RJ, Beher WT. Bile acid-mediated postcholecystectomy diarrhea. Arch Intern Med. 1987; 147 (7): 1327-1329.

19. Schiller LR. Secretory diarrhea. Curr Gastroenterol Rep. 1999;1: 389-397.

20. Ruiz-Campos L, Gisbert JP, Ysamat M, Arau B, Loras C, Esteve M et al. Systematic review with meta-analysis: the prevalence of bile acid malabsorption and response to colestyramine in patients with chronic watery diarrhoea and previous cholecystectomy. Aliment Pharmacol Ther. 2019; 49 (3): 242-250.

21. Mertens MC, De Vries J, Scholtes VP, Jansen P, Roukema JA. Prospective 6 weeks follow-up post-cholecystectomy: the predictive value of pre-operative symptoms. J Gastrointest Surg. 2009; 13 (2): 304-311.

22. Berger MY, Olde Hartman TC, Bohnen AM. Abdominal symptoms: do they disappear after cholecystectomy? Surg Endosc. 2003; 17 (11): 1723-1728.

23. Bisgaard T, Rosenberg J, Kehlet H. From acute to chronic pain after laparoscopic cholecystectomy: a prospective follow-up analysis. Scand J Gastroenterol. 2005; 40 (11): 1358-1364.

24. Zhang J, Lu Q, Ren YF, Dong J, Mu YP, Lv Y et al. Factors relevant to persistent upper abdominal pain after cholecystectomy. HPB (Oxford). 2017; 19 (7): 629-637.

25. McNally MA, Locke GR, Zinsmeister AR, Schleck CD, Peterson J, Talley NJ. Biliary events and an increased risk of new onset irritable bowel syndrome: a population-based cohort study. Aliment Pharmacol Ther. 2008; 28 (3): 334-343.

26. Farahmandfar MR, Chabok M, Alade M, Bouhelal A, Patel B. Post cholecystectomy diarrhoea - a systematic review. Surg Sci. 2012; 3 (6): 332-338.

27. Amieva-Balmori M, Azamar-Jácome AA, Rojas-Carrera SI, CanoContreras AD, Remes-Troche JM. Prevalence of irritable bowel syndrome in cholecystectomized patients. Is there any association? Med Int Mex. 2016; 32 (2): 161-168.

28. Fall K, Ye W, Nyrén O. Risk for gastric cancer after cholecystectomy. Am J Gastroenterol. 2007; 102 (6): 1180-1184.

29. Aprea G, Canfora A, Ferronetti A, Giugliano A, Guida F, Braun A et al. Morpho-functional gastric pre-and post-operative changes in elderly patients undergoing laparoscopic cholecystectomy for gallstone related disease. BMC Surg. 2012; 12 Suppl 1: S5.

30. Mercan E, Duman U, Tihan D, Dilektasli E, Senol K. Cholecystectomy and duodenogastric reflux: interacting effects over the gastric mucosa. Springerplus. 2016; 5 (1): 1970.

31. Ozkaya M, Erten A, Sahin I, Engin B, Ciftçi A, Cakal E et al. The effect of ursodeoxycholic acid treatment on epidermal growth factor in patients with bile reflux gastritis. Turk J Gastroenterol. 2002; 13 (4): 198-202.

32. Zaniewski M, Ziaja K, Nowakowski P, Zabski M, Kuszewski M. Is a post-cholecystectomy syndrome the result of inappropriate preoperative diagnosis? Wiad Lek. 1999; 52 (11-12): 587-590.

33. Isherwood J, Oakland K, Khanna A. A systematic review of the aetiology and management of post cholecystectomy syndrome. Surgeon. 2019; 17 (1): 33-42.

34. Peterli R, Schuppisser JP, Herzog U, Ackermann C, Tondelli PE. Prevalence of postcholecystectomy symptoms: long-term outcome after open versus laparoscopic cholecystectomy. World J Surg. 2000; 24 (10): 1232-1235. 